\title{
FROBENIUS-SCHUR INDICATORS OF UNIPOTENT CHARACTERS AND THE TWISTED INVOLUTION MODULE
}

\author{
MEINOLF GECK AND GUNTER MALLE
}

\begin{abstract}
Let $W$ be a finite Weyl group and $\sigma$ a non-trivial graph automorphism of $W$. We show a remarkable relation between the $\sigma$-twisted involution module for $W$ and the Frobenius-Schur indicators of the unipotent characters of a corresponding twisted finite group of Lie type. This extends earlier results of Lusztig and Vogan for the untwisted case and then allows us to state a general result valid for any finite group of Lie type. Inspired by recent work of Marberg, we also formally define Frobenius-Schur indicators for "unipotent characters" of twisted dihedral groups.
\end{abstract}

\section{INTRODUCTION}

Let $G$ be a connected reductive algebraic group over $\overline{\mathbb{F}}_{p}$ (where $p$ is a prime) and $F: G \rightarrow G$ an endomorphism such that some power of $F$ defines a rational structure on $G$ relative to a finite subfield of $\overline{\mathbb{F}}_{p}$. Then the fixed point set $G^{F}$ is a finite group of Lie type. Let $W$ be the Weyl group of $G$ and $S$ a corresponding set of simple reflections, defined with respect to an $F$-stable maximal torus contained in an $F$-stable Borel subgroup of $G$. Let $\operatorname{Uch}\left(G^{F}\right)$ be the set of unipotent characters, as defined by Deligne and Lusztig [2]. An irreducible character $\chi$ of $G^{F}$ is unipotent if and only if $\chi$ appears with non-zero multiplicity in some Deligne-Lusztig virtual character $R_{T_{w}, 1}$ where $T_{w}$ is an $F$-stable maximal torus "of type $w$ " and 1 stands for the trivial character of $T_{w}^{F}$.

Assuming that $G$ is of split type, Lusztig and Vogan [16] have established a connection between the Frobenius-Schur indicators of the unipotent characters of $G^{F}$ and a certain involution module for $W$ which appeared in the work of Kottwitz [6]. More precisely, let $\mathrm{CF}_{0}\left(G^{F}\right)$ be the space of all uniform unipotent class functions on $G^{F}$, that is, the subspace of the space of class functions on $G^{F}$ which is spanned by all the virtual characters $R_{T_{w}, 1}$. For any class function $\psi$ on $G^{F}$, denote by $\psi_{0}$ the orthogonal projection onto the subspace $\mathrm{CF}_{0}\left(G^{F}\right)$. Then, by [16, 6.4], we have the following remarkable identity (assuming that $G$ is of split type):

$$
\left(\sum_{\chi \in \operatorname{Uch}\left(G^{F}\right)} \nu(\chi) \chi\right)_{0}=\frac{1}{|W|} \sum_{w \in W} \rho(w) R_{T_{w}, 1} ;
$$

here, $\nu(\chi)$ denotes the Frobenius-Schur indicator of $\chi$ and $\rho$ is the character of Kottwitz' involution module of $W$. In this paper, we extend this to the situation where $W$ is not necessarily of split type. Then $F$ induces a non-trivial automorphism $\sigma: W \rightarrow W$ such that $\sigma(S)=S$. We shall consider a canonical extension of the involution module to the semidirect product $\tilde{W}=W \rtimes\langle\sigma\rangle$; let $\tilde{\rho}$ be the

Received by the editors April 22, 2012 and, in revised form, October 10, 2012.

2010 Mathematics Subject Classification. Primary 20C15; Secondary 20C33. 
character of this extended module. Then we shall show that the above identity remains true where, on the right-hand side, the term $\rho(w)$ needs to be replaced by $\tilde{\rho}(w \sigma)$. With this modification, we actually obtain a statement valid for any $G^{F}$; see Corollary 5.3 .

The proof of this result proceeds as in [16], by reformulating the desired identity in terms of Lusztig's Fourier transform [10, Chap. 4] (where we use the Fourier matrices in [4] for the Suzuki and Ree groups). Our main result, Theorem 5.1, shows that as in the split case, the multiplicities in the decomposition of $\tilde{\rho}$ as a class function on the coset $W . \sigma$ are obtained via Fourier transform from the FrobeniusSchur indicators of the unipotent characters of $G^{F}$. We prove Theorem 5.1 by explicitly decomposing $\tilde{\rho}$ in all relevant cases (see Section 3 for classical types and Section 4 for exceptional types) and then comparing with the Fourier transform of the vector of Frobenius-Schur indicators in Section 5 The main difficulty is of a somewhat technical nature: in the twisted case, both the Fourier matrices and the formulae for the decomposition of $\tilde{\rho}$ essentially rely on choices of extensions of $\sigma$-stable irreducible characters of $W$ and, a priori, there is no reason why the choices on both sides should fit together. We shall see that the formulae for the decomposition of $\tilde{\rho}$ become particularly simple when we choose Lusztig's preferred extensions, as defined in [11, 17.2]. Note also that in the split case all FrobeniusSchur indicators of unipotent characters are 0 or 1 , which is no longer the case in our setting. Already in type ${ }^{2} A_{n}$, it is quite remarkable how the multiplicities in the decomposition of $\tilde{\rho}$ match the distribution of the \pm 1 values of the Frobenius-Schur indicators of the unipotent characters of $\mathrm{GU}_{n}(q)$.

Finally, the construction of $\tilde{\rho}$ also works for the case where $W$ is any dihedral group and $\sigma \neq \mathrm{id}$. Thus, inspired by Marberg [17, our computations also allow us to formally define Frobenius-Schur indicators for "unipotent characters" of twisted dihedral groups; see Theorem 5.4 .

\section{The EXtended involution MOdule}

Let $(W, S)$ be a finite Coxeter group, with distinguished set of generators $S$. We write $\mathbf{I}:=\left\{w \in W \mid w^{2}=1\right\}$ for the subset of elements of order at most 2 . Let $\mathcal{H}(W)$ be the one-parameter Iwahori-Hecke algebra over $\mathbb{Q}\left[v^{ \pm 1}\right]$ attached to $W$ with parameter $v^{2}$, with standard basis $\left\{T_{w} \mid w \in W\right\}$. (This basis is normalised such that the quadratic relations read $\left(T_{s}-v^{2}\right)\left(T_{s}+1\right)=0$ for $s \in S$.) In [14], Lusztig shows that an action of $\mathcal{H}(W)$ on a free $\mathbb{Q}\left[v^{ \pm 1}\right]$-module with basis $\left\{a_{w} \mid w \in \mathbf{I}\right\}$ can be defined by the following formulae:

$$
T_{s} \cdot a_{w}= \begin{cases}v a_{w}+(v+1) a_{s w} & \text { if } s w=w s, l(w s)>l(w), \\ \left(v^{2}-v-1\right) a_{w}+\left(v^{2}-v\right) a_{s w} & \text { if } s w=w s, l(w s)<l(w), \\ a_{s w s} & \text { if } s w \neq w s, l(w s)>l(w), \\ \left(v^{2}-1\right) a_{w}+v^{2} a_{s w s} & \text { if } s w \neq w s, l(w s)<l(w),\end{cases}
$$

for $s \in S, w \in \mathbf{I}$. We write $R_{v}$ for this representation of $\mathcal{H}(W)$. As in [16, 6.3], this representation induces a representation $R$ of $W$ on the $\mathbb{Q}$-vector space $V=$ $\bigoplus_{w \in \mathbf{I}} \mathbb{Q} a_{w}$, defined by

$$
R(s) . a_{w}:= \begin{cases}-a_{w} & \text { if } s w=w s, l(w s)<l(w) \\ a_{s w s} & \text { else. }\end{cases}
$$


Let $C$ be a conjugacy class of $W$ contained in $\mathbf{I}$. Then it is clear that the subspace $V_{C}:=\left\langle a_{w} \mid w \in C\right\rangle \subseteq V$ is a submodule; furthermore, we have a direct sum decomposition $V=\bigoplus_{C} V_{C}$ where $C$ runs over the conjugacy classes of $W$ contained in I. Now let us fix such a conjugacy class $C$. Then an alternative description of $V_{C}$ is given as follows.

By [5. 3.2.10], $C$ contains an element of the form $w_{I}$ where $I \subseteq S$ and $w_{I}$ is the longest element in the parabolic subgroup $W_{I} \subseteq W$; furthermore, $w_{I}$ is central in $W_{I}$. By [5, 2.1.15], we have a semidirect product decomposition $N_{W}\left(W_{I}\right)=Y \ltimes W_{I}$ where $Y$ is a group consisting of certain distinguished left coset representatives of $W_{I}$ in $W$ such that $I$ is invariant under conjugation with all $y \in Y$; in particular, we have $l\left(y w^{\prime}\right)=l(y)+l\left(w^{\prime}\right)$ for all $y \in Y$ and $w^{\prime} \in W_{I}$. Let $\varepsilon_{I}: N_{W}\left(W_{I}\right) \rightarrow\{ \pm 1\}$ be the trivial extension of the sign representation of $W_{I}$, that is, we have

$$
\varepsilon_{I}\left(y w^{\prime}\right)=(-1)^{l\left(w^{\prime}\right)} \quad \text { for all } y \in Y \text { and } w^{\prime} \in W_{I} .
$$

Lemma 2.1. In the above setting, we have

$$
N_{W}\left(W_{I}\right)=C_{W}\left(w_{I}\right) \quad \text { and } \quad V_{C} \cong \operatorname{Ind}_{C_{W}\left(w_{I}\right)}^{W}\left(\varepsilon_{I}\right) .
$$

Proof. First we show the equality $N_{W}\left(W_{I}\right)=C_{W}\left(w_{I}\right)$. Let $w \in N_{W}\left(W_{I}\right)$. We write $w=y w^{\prime}$ where $y \in Y$ and $w^{\prime} \in W_{I}$. Since $y I y^{-1}=I$, we have $y w_{I} y^{-1}=w_{I}$. Since $w_{I}$ is central in $W_{I}$, we conclude that $w=y w^{\prime} \in C_{W}\left(w_{I}\right)$. Conversely, let $w \in$ $C_{W}\left(w_{I}\right)$. We write $w=x w^{\prime}$ where $w^{\prime} \in W_{I}$ and $x$ is some distinguished left coset representative of $W_{I}$ in $W$. Since $w^{\prime}$ commutes with $w_{I}$, we also have $x w_{I}=w_{I} x$. Since $l\left(x w_{I}\right)=l(x)+l\left(w_{I}\right)$, we conclude that $l\left(x^{-1} w_{I}\right)=l\left(w_{I} x\right)=l(x)+l\left(w_{I}\right)$ and so both $x$ and $x^{-1}$ are distinguished left coset representatives. Hence, by [5, 2.1.12], we have $x W_{I} x^{-1} \cap W_{I}=W_{J}$ where $J=x I x^{-1} \cap I$. But $w_{I}=x w_{I} x^{-1}$ lies in this intersection and, hence, in $W_{J}$. It follows that $I=J$ and so $x \in N_{W}\left(W_{I}\right)$.

To prove the statement concerning $V_{C}$, it is sufficient to show that

$$
\left(y w^{\prime}\right) \cdot a_{w_{I}}=(-1)^{l\left(w^{\prime}\right)} a_{w_{I}} \quad \text { for all } y \in Y \text { and } w^{\prime} \in W_{I} .
$$

This is seen as follows. For any $s \in I$, we have $s . a_{w_{I}}=-a_{w_{I}}$. Consequently, we have $w^{\prime} \cdot a_{w}=(-1)^{l\left(w^{\prime}\right)} a_{w_{I}}$. Thus, it remains to show that $y \cdot a_{w_{I}}=a_{w_{I}}$ for all $y \in Y$. We shall in fact show that $x \cdot a_{w_{I}}=a_{x w_{I} x^{-1}}$ where $x$ is any distinguished left coset representative of $W_{I}$ in $W$. We proceed by induction on $l(x)$. If $x=1$, the assertion is clear. Now assume that $x \neq 1$ and choose $s \in S$ such that $l(s x)<l(x)$. By Deodhar's Lemma [5, 2.1.2], we also have that $z:=s x$ is a distinguished left coset representative. Hence, using induction, we have $z \cdot a_{w_{I}}=a_{z w_{I} z^{-1}}$ and so

$$
x . a_{w_{I}}=s . a_{z w_{I} z^{-1}} .
$$

Given the formula for the action of a generator on the basis elements of $V_{C}$, it now suffices to show that $s$ does not commute with $z w_{I} z^{-1}$ or that $l\left(z w_{I} z^{-1} s\right)>$ $l\left(z w_{I} z^{-1}\right)$. Assume, if possible, that none of these two conditions is satisfied, that is, we have that $s$ commutes with $z w_{I} z^{-1}$ and $l\left(s z w_{I} z^{-1}\right)=l\left(z w_{I} z^{-1} s\right)<$ $l\left(z w_{I} z^{-1}\right)$. Then the "Exchange Lemma" (see [5, Exc. 1.6]) and the fact that $l\left(s z w_{I}\right)=l(z)+l\left(w_{I}\right)+1$ imply that $s z w_{I} z^{-1}=z w_{I} u$ where $l(u)<l(z)$. Since $s$ commutes with $z w_{I} z^{-1}$, we have $z w_{I} z^{-1} s=s z w_{I} z^{-1}=z w_{I} u$ and so $z^{-1} s=u$. This would imply that $l(u)=l\left(z^{-1} s\right)=l(s z)>l(z)$, a contradiction. Hence, the assumption was wrong and so $x . a_{w_{I}}=a_{x w_{I} x^{-1}}$, as required.

Remark 2.2. It is stated in [16, 6.3] that the $W$-module $V$ is isomorphic to the involution module constructed by Kottwitz [6]. Lemma 2.1 provides an easy proof 
of this statement. Indeed, assume that $W$ is realised as a subgroup generated by reflections in $\operatorname{GL}(E)$, where $E$ is a finite-dimensional Euclidean space. Let $\Phi$ be the corresponding root system and let $\Phi^{+}$be the set of positive roots defined by $S$. Let $t \in \mathbf{I}$. Following Kottwitz [6], a root $\alpha \in \Phi$ is called imaginary with respect to $t$ if $t(\alpha)=-\alpha$. Then Kottwitz defines a linear representation $\delta: C_{W}(t) \rightarrow\{ \pm 1\}$ such that $\delta(w)=(-1)^{k}$ where $k$ is the number of positive imaginary roots which are sent to negative roots by $w$. Now, if $t=w_{I}$ (where $w_{I}$ is central in $W_{I}$ as above), then one easily sees that the imaginary roots with respect to $w_{I}$ are precisely the roots in the parabolic subsystem $\Phi_{I} \subseteq \Phi$ corresponding to $W_{I}$. Furthermore, let $w \in C_{W}\left(w_{I}\right)$ and write $w=y w^{\prime}$ where $y \in Y$ and $w^{\prime} \in W_{I}$. Then $l\left(w^{\prime}\right)$ is the number of positive roots in $\Phi_{I}$ which are sent to negative roots by $w^{\prime}$. Since $y$ sends all positive roots in $\Phi_{I}$ to positive roots, we conclude that $l\left(w^{\prime}\right)$ is the number of positive imaginary roots sent to negative roots by $w$. Thus, we have $\delta=\varepsilon_{I}$, which yields the isomorphism between $V$ and the involution module constructed by Kottwitz.

Now let $\sigma \in \operatorname{Aut}(W)$ be an automorphism of $W$ as a Coxeter group, that is, $\sigma$ stabilizes the set $S$ of distinguished generators. In particular, $\sigma$ preserves lengths in $W$. Then there is a natural action of $\sigma$ on the Iwahori-Hecke algebra $\mathcal{H}(W)$, and we obtain the extended Hecke algebra $\tilde{\mathcal{H}}(W)$, the associative $\mathbb{Q}\left[v^{ \pm 1}\right]$-algebra generated by $\mathcal{H}(W)$ together with an additional element $T_{\sigma}$ subject to the relations $T_{\sigma}^{d}=1$ and $T_{\sigma} T_{s}=T_{\sigma(s)} T_{\sigma}$ for all $s \in S$, where $d=o(\sigma)$ denotes the order of $\sigma$. With this we have the following 1 :

Proposition 2.3. The above representation $R_{v}$ of $\mathcal{H}(W)$ extends to a representation $\tilde{R}_{v}$ of $\tilde{\mathcal{H}}(W)$ via

$$
T_{\sigma} . a_{w}:=a_{\sigma(w)} \quad \text { for all } w \in \mathbf{I} .
$$

Proof. It suffices to check that the additional relations of $\tilde{\mathcal{H}}(W)$ involving $T_{\sigma}$ are respected. It is clear that $T_{\sigma}^{d}$ acts as the identity. Furthermore, for $s \in S$ with $\sigma(s)=s^{\prime}$ we have

$$
\begin{aligned}
T_{\sigma} T_{s} \cdot a_{w} & =\left\{\begin{array}{ll}
v a_{\sigma(w)}+(v+1) a_{s^{\prime} \sigma(w)} & \text { if } s w=w s, l(w s)>l(w) \\
\left(v^{2}-v-1\right) a_{\sigma(w)}+\left(v^{2}-v\right) a_{s^{\prime} \sigma(w)} & \text { if } s w=w s, l(w s)<l(w) \\
a_{s^{\prime} \sigma(w) s^{\prime}} & \text { if } s w \neq w s, l(w s)>l(w) \\
\left(v^{2}-1\right) a_{\sigma(w)}+v^{2} a_{s^{\prime} \sigma(w) s^{\prime}} & \text { if } s w \neq w s, l(w s)<l(w)
\end{array}\right\} \\
& =T_{s^{\prime}} T_{\sigma} \cdot a_{w}
\end{aligned}
$$

for all $w \in \mathbf{I}$, since $\sigma$ leaves the length function invariant.

Let $\tilde{W}$ be the semidirect product of $W$ with $\langle\sigma\rangle$. Thus, $\tilde{W}=\langle W, \sigma\rangle$ and, in $\tilde{W}$, we have the identity $\sigma(w)=\sigma w \sigma^{-1}$ for all $w \in W$. By an argument similar to that in the proof of Proposition 2.3, we obtain:

Proposition 2.4. The representation $R$ of $W$ extends to a representation $\tilde{R}$ of $\tilde{W}=\langle W, \sigma\rangle$ via

$$
\tilde{R}(\sigma) . a_{w}:=a_{\sigma(w)} \quad \text { for all } w \in \mathbf{I} \text {. }
$$

\footnotetext{
${ }^{1}$ This result also appears in the first version of a recent preprint [15] of Lusztig.
} 
Let us write $\rho$ for the character of the representation $R$, and similarly $\tilde{\rho}$ for that of $\tilde{R}$. We are interested in the decomposition of $\tilde{\rho}$ as a class function on the coset $W . \sigma$. In the case that $\sigma=\mathrm{id}$, this decomposition was obtained by Kottwitz [6], Casselman [1] for Weyl groups and by Marberg [17] for the non-crystallographic Coxeter groups. Here, we discuss the case when $\sigma \neq \mathrm{id}$.

Remark 2.5. Assume that $C \subseteq \mathbf{I}$ is a $\sigma$-stable conjugacy class of $W$. Then $V_{C}$ is a $\tilde{W}$-submodule of $V$ and

$$
\tilde{\rho}_{C}:=\left.\tilde{\rho}\right|_{V_{C}}=\operatorname{Ind}_{C_{\tilde{W}}(t)}^{\tilde{W}}\left(\tilde{\epsilon}_{C}\right) \quad \text { for any } t \in C,
$$

where $\tilde{\epsilon}_{C}: C_{\tilde{W}}(t) \rightarrow\{ \pm 1\}$ is the linear character defined by $\tilde{R}(w) \cdot a_{t}=\tilde{\epsilon}_{C}(w) a_{t}$ for $w \in C_{\tilde{W}}(t)$. On the other hand, if $C \subseteq \mathbf{I}$ is a conjugacy class which is not $\sigma$-invariant, then the trace of any element in $W \cdot \sigma$ on $\sum_{i=1}^{d} V_{\sigma^{i}(C)}$ is zero. Thus, as a class function on $W . \sigma$, we have

$$
\tilde{\rho}=\sum_{C} \tilde{\rho}_{C}
$$

where the sum runs over all $\sigma$-stable conjugacy classes $C$ of $W$ which are contained in I. Now let $C$ be such a conjugacy class. By Frobenius reciprocity, we have

$$
\left\langle\tilde{\rho}_{C}, \operatorname{Ind}_{W}^{\tilde{W}}(\phi)\right\rangle=\left\langle\rho_{C}, \phi\right\rangle \quad \text { for all } \phi \in \operatorname{Irr}(W) .
$$

Assume further that $d=o(\sigma)$ is a prime number (which will be the case in all situations we shall consider). If $\phi^{\sigma} \neq \phi$, then $\operatorname{Ind}_{W}^{\tilde{W}}(\phi)$ is irreducible; otherwise, $\operatorname{Ind}_{W}^{\tilde{W}}(\phi)$ is the sum of $d$ distinct extensions of $\phi$ to $\tilde{W}$. Hence, as a class function on $W . \sigma$, we have a unique decomposition

$$
\tilde{\rho}_{C}=\sum_{\phi \in \operatorname{Irr}(W)^{\sigma}} n_{\phi} \tilde{\phi} \quad\left(n_{\phi} \in \mathbb{Z}[\sqrt[d]{1}]\right),
$$

where each $\tilde{\phi}$ is a fixed extension of $\phi \in \operatorname{Irr}(W)^{\sigma}$ to the coset W. $\sigma$. So the problem will be to fix choices for the extensions $\tilde{\phi}$ and to determine the corresponding coefficients $n_{\phi}$.

In order to deal with the various possibilities for $W, \sigma$ where $W$ is irreducible, the following general result will be useful. Assume that $\sigma \neq$ id acts as an inner automorphism of $W$. Since the only non-trivial element of $W$ fixing $S$ is the longest element $w_{0}$ of $W$, this implies that $\sigma$ acts by conjugation with $w_{0}$. By the classification of finite Coxeter groups, $W$ is of type $A_{n}, D_{2 n+1}, I_{2}(2 m+1)$ or $E_{6}$. Note that in all of these groups, the centralizer of $\sigma$ contains a Sylow 2-subgroup of $W$, so fixes some element in any conjugacy class $C \subseteq \mathbf{I}$. Note also that, in these cases, the element $w_{0} \sigma$ lies in the center of $\tilde{W}$ and so $\tilde{\phi}\left(w_{0} \sigma\right)= \pm \phi(1)$ for every extension $\tilde{\phi}$ of $\phi \in \operatorname{Irr}(W)^{\sigma}$.

Proposition 2.6. Assume that $\sigma$ acts on $W$ by conjugation with $w_{0}$. Let $C \subseteq \mathbf{I}$ be a $\sigma$-stable conjugacy class. Then, as a class function on $W . \sigma$, we have

$$
\tilde{\rho}_{C}=\sum_{\phi \in \operatorname{Irr}(W)}\left\langle\rho_{C}, \phi\right\rangle \tilde{\phi},
$$

where $\tilde{\phi}$ denotes the (unique) extension of $\phi$ to W. $\sigma$ such that $(-1)^{l(t)} \tilde{\phi}\left(w_{0} \sigma\right)>0$, for any $t \in C$. In particular, $\phi\left(w_{0}\right)$ has the same sign for all constituents $\phi$ of $\rho_{C}$ with $\phi\left(w_{0}\right) \neq 0$. 
Proof. By assumption, $w_{0} \sigma$ lies in the center of $\tilde{W}$, so it acts by the scalar $\epsilon_{C}\left(w_{0} \sigma\right)$ in the representation $\tilde{R}_{C}$. But $w_{0}$ sends all positive roots to negative ones while $\sigma$ stabilizes the set of positive roots, so by the explicit formula for $\epsilon_{C}$ above we have that $\epsilon_{C}\left(w_{0} \sigma\right)=(-1)^{l(t)}$. In particular, $\psi\left(w_{0} \sigma\right)=(-1)^{l(t)} \psi(1) \neq 0$ for any constituent $\psi \in \operatorname{Irr}(\tilde{W})$ of $\tilde{\rho}_{C}$, whence $\left\langle\rho_{C}, \phi\right\rangle=\left\langle\tilde{\rho}_{C}, \tilde{\phi}\right\rangle$ for the extension $\tilde{\phi}$ of $\phi$ given in the statement. Since $\tilde{R}_{C}(\sigma)$ is a permutation matrix, it is then clear that $\tilde{\rho}_{C}\left(w_{0}\right)=\rho_{C}\left(w_{0}\right)$ has the same sign as $\tilde{\rho}_{C}\left(w_{0} \sigma\right)$. Thus for all constituents $\phi$ of $\rho_{C}$ with $\phi\left(w_{0}\right) \neq 0, \phi\left(w_{0}\right)$ has the same sign $(-1)^{l(t)}$.

Remark 2.7. Let $\phi \in \operatorname{Irr}(W)$ be such that $\phi^{\sigma}=\phi$. In [11, 17.2], Lusztig has defined the notion of a preferred extension $\tilde{\phi}$ of $\phi$ to $\tilde{W}$. In the cases where $W$ is an irreducible Weyl group and $\sigma$ is "ordinary" in the sense of [10, 3.1] (that is, whenever $s \neq s^{\prime}$ in $S$ are in the same $\sigma$-orbit, then the product $s s^{\prime}$ has order 2 or 3 ), these are given as follows.

- If $\sigma=$ id, then $\tilde{\phi}=\phi$.

- If $\sigma$ acts by conjugation with $w_{0}$ and $W$ is of type $A_{n}(n \geq 2)$ or $E_{6}$, then $\tilde{\phi}$ is the unique extension such that $\tilde{\phi}\left(w_{0} \sigma\right)=(-1)^{a_{\phi}} \phi(1)$ where $a_{\phi}$ is the invariant defined in [10,4.1] (in terms of the generic degree polynomial associated with $\phi$ ).

- If $\sigma$ has order 3 and $W$ is of $D_{4}$, then $\tilde{\phi}$ is the unique extension which is defined over $\mathbb{Q}($ see $[10,3.2])$.

- If $\sigma$ has order 2 and $W$ is of type $D_{n}(n \geq 4)$, then $\tilde{\phi}$ is defined as follows. In this case, $\tilde{W}$ can be identified with a Weyl group of type $B_{n}$ and, as in [10. 4.18], the irreducible characters of $\tilde{W}$ which remain irreducible upon restriction to $W$ are parametrised by certain symbols with two rows of equal length (an upper row and a lower row). Then $\tilde{\phi} \in \operatorname{Irr}(\tilde{W})$ is the preferred extension of $\phi$ if the corresponding symbol has the following property: the smallest entry which appears in only one row appears in the lower row.

\section{TYPES $A_{n}$ AND $D_{n}$}

In this and the following section we explicitly determine the decomposition of $\tilde{\rho}$ (as a class function on $W . \sigma$, see Remark 2.5), in all cases where $W$ is irreducible and $\sigma \neq$ id.

3.1. We begin with the case where $W=\mathfrak{S}_{n}$. Here, the irreducible characters of $W$ are labelled by partitions $\alpha \vdash n$, and we write $\phi_{\alpha}$ for the corresponding character. The non-trivial $\sigma$ is given by conjugation with the longest element $w_{0}$.

Proposition 3.1. Let $W=\mathfrak{S}_{n}$ and let $\sigma$ be the graph automorphism of order 2 . As a class function on $W . \sigma$, we have

$$
\tilde{\rho}=\sum_{\alpha \vdash n} \tilde{\phi}_{\alpha}
$$

where $\tilde{\phi}_{\alpha}$ denotes Lusztig's preferred extension as in Remark 2.7.

Proof. Let $\alpha \vdash n$ and denote by $m_{\alpha}$ the number of odd parts of the conjugate partition $\alpha^{\prime}$. By [6, 3.1], $\phi_{\alpha}$ is a constituent of $\rho_{C}$, where $C$ is the class of involutions of $\mathfrak{S}_{n}$ with exactly $m_{\alpha}$ fixed points. Now clearly there is such an involution $t$ of length $\left(n-m_{\alpha}\right) / 2$, whence $\epsilon_{C}\left(w_{0} \sigma\right)=(-1)^{l(t)}=(-1)^{\left(n-m_{\alpha}\right) / 2}$. Thus, by Proposition 2.6 we have $\tilde{\rho}=\sum_{\alpha \vdash n} \tilde{\phi}_{\alpha}$ where $\tilde{\phi}_{\alpha}$ is the unique extension such that 
$\tilde{\phi}_{\alpha}\left(w_{0} \sigma\right)=(-1)^{\left(n-m_{\alpha}\right) / 2} \phi_{\alpha}(1)$. Finally, let $\alpha \vdash n$ and denote by $\left(\alpha_{1}^{\prime}, \ldots, \alpha_{r}^{\prime}\right)$ the parts of the conjugate partition $\alpha^{\prime}$. Then by [5, 5.4.1] we have

$$
2 a_{\chi} \equiv \sum_{1 \leq i \leq r} \alpha_{i}^{\prime}\left(\alpha_{i}^{\prime}-1\right)=\sum_{1 \leq i \leq r} \alpha_{i}^{\prime 2}-n \equiv m_{\alpha}-n \quad(\bmod 4) .
$$

This shows that $\left(n-m_{\alpha}\right) / 2 \equiv a_{\alpha}(\bmod 4)$ and so the extension $\tilde{\phi}$ chosen above is Lusztig's preferred extension.

3.2. For the remainder of this section, let $W$ be of type $D_{n}(n \geq 2)$ and $\sigma$ a graph automorphism of order 2. In this case, the notation can be arranged such that $\tilde{W}=\langle W, \sigma\rangle$ is a Coxeter group of type $B_{n}$ with generating set $S=\left\{\sigma, s_{1}, \ldots, s_{n-1}\right\}$ and diagram:

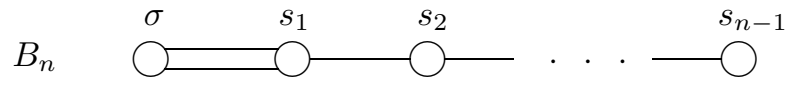

Furthermore, $s_{1}, \ldots, s_{n-1}$ together with $u:=\sigma s_{1} \sigma$ are Coxeter generators for $W$ where $u, s_{1}$ commute with each other. As in [5, §5.5], we have a labelling of the irreducible characters of $\tilde{W}$ by pairs of partitions $(\alpha, \beta)$ such that $|\alpha|+|\beta|=n$. We write this as

$$
\operatorname{Irr}(\tilde{W})=\left\{\tilde{\phi}^{\alpha, \beta} \mid(\alpha, \beta) \vdash n\right\} ;
$$

for example, the pair $((n),-)$ labels the trivial character and $\left(-,\left(1^{n}\right)\right)$ labels the sign character. Let $(\alpha, \beta) \vdash n$ and denote by $\phi^{\alpha, \beta}$ the restriction of $\tilde{\phi}^{\alpha, \beta}$ from $\tilde{W}$ to $W$. It is well known that $\phi^{\alpha, \beta}=\phi^{\beta, \alpha} \in \operatorname{Irr}(W)$ if $\alpha \neq \beta$ and $\phi^{\alpha, \alpha}=\phi^{\alpha,+}+\phi^{\alpha,-}$ where $\phi^{\alpha, \pm} \in \operatorname{Irr}(W)$. Furthermore, all irreducible characters of $W$ arise in this way.

Remark 3.2. In the above setting, let $C \subseteq W$ be a conjugacy class of involutions which is invariant under $\sigma$. Then there exists a parabolic subgroup $\tilde{W}_{I} \subseteq \tilde{W}$, where $I \subseteq\left\{\sigma, s_{1}, \ldots, s_{n-1}\right\}$, such that the following conditions are satisfied:

$(*)$ The longest element $w_{I} \in \tilde{W}_{I}$ is central in $\tilde{W}_{I}$ and we have $w_{I} \in C$.

This is seen as follows. By [5, 3.2.10], $C$ contains an element of the form $w_{I^{\prime}}$ where $I^{\prime} \subseteq\left\{u, s_{1}, \ldots, s_{n-1}\right\}$ and $w_{I^{\prime}}$ is the longest element in the parabolic subgroup $W_{I^{\prime}} \subseteq W$; furthermore, $w_{I^{\prime}}$ is central in $W_{I^{\prime}}$. If both $u$ and $s_{1}$ belong to $I^{\prime}$, then we certainly have $w_{I^{\prime}}=w_{I}$ where $I \subseteq\left\{\sigma, s_{1}, \ldots, s_{n-1}\right\}$ is the subset obtained by replacing $u$ by $\sigma$ in $I^{\prime}$. Then $(*)$ holds. Otherwise, since $C$ is invariant under $\sigma$, we can assume, without loss of generality, that $I^{\prime} \subseteq\left\{s_{1}, s_{2}, \ldots, s_{n-1}\right\}$. Then we can set $I=I^{\prime}$. Again, $(*)$ holds.

In the first case, it is automatically true that $\sigma\left(w_{I}\right)=w_{I}$. In the second case, using [5, 3.4.12], we can even assume that $I^{\prime} \subseteq\left\{s_{2}, s_{3}, \ldots, s_{n-1}\right\}$ and then set $I=I^{\prime}$. Hence, in both cases, $I$ can actually be chosen such that $\sigma\left(w_{I}\right)=w_{I}$.

Remark 3.3. Let $C \subseteq W$ and $I \subseteq\left\{\sigma, s_{1}, \ldots, s_{n-1}\right\}$ be as in Remark 3.2. We obtain the corresponding $W$-module $V_{C}$ (as constructed in Section 2), with character given by

$$
\rho_{C}=\operatorname{Ind}_{C_{W}\left(w_{I}\right)}^{W}\left(\epsilon_{I}\right)
$$

where $\epsilon_{I}: C_{W}\left(w_{I}\right) \rightarrow\{ \pm 1\}$ is a certain linear character of $C_{W}\left(w_{I}\right)$; see Lemma 2.1. Let $\tilde{\rho}_{C}$ be the extension of $\rho_{C}$ to $\tilde{W}$. Now, as in Section 2], we have $C_{\tilde{W}}\left(w_{I}\right)=$ $N_{\tilde{W}}\left(\tilde{W}_{I}\right)=\tilde{W}_{I} \rtimes \tilde{Y}$ where $\tilde{Y}$ is a group consisting of certain distinguished left coset 
representatives of $\tilde{W}_{I}$ in $\tilde{W}$. Let $\tilde{\epsilon}_{I}: C_{\tilde{W}}\left(w_{I}\right) \rightarrow\{ \pm 1\}$ be the linear character such that

$$
\tilde{\epsilon}_{I}\left(y w^{\prime}\right)=(-1)^{l\left(w^{\prime}\right)-l_{\sigma}\left(w^{\prime}\right)} \quad \text { for all } y \in \tilde{Y} \text { and } w^{\prime} \in \tilde{W}_{I},
$$

where $l_{\sigma}(w)$ denotes the number of occurrences of the generator $\sigma$ in a reduced expression for $w^{\prime} \in \tilde{W}$. Note that $\tilde{\epsilon}_{I}$ is an extension of the linear character $\epsilon_{I}$ of $C_{W}\left(w_{I}\right)$ which is used in the construction of $\rho_{C}$. Then we have

$$
\tilde{\rho}_{C}=\operatorname{Ind}_{C_{\tilde{W}}\left(w_{I}\right)}^{\tilde{W}}\left(\tilde{\epsilon}_{I}\right) \text {. }
$$

(This is seen by an argument entirely analogous to that in Lemma 2.1.)

Lemma 3.4. In the above setting, we have $\left\langle\tilde{\phi}^{\alpha, \beta}, \tilde{\rho}_{C}\right\rangle=0$ unless $|\alpha|>|\beta|$.

Proof. First recall that $\tilde{W}$ has a normal subgroup $\tilde{N}=\left\langle\sigma_{1}, \sigma_{2}, \ldots, \sigma_{n}\right\rangle$ where $\sigma_{1}:=$ $\sigma$ and $\sigma_{i}:=s_{i-1} \sigma_{i-1} s_{i-1}$ for $2 \leq i \leq n$. Let $0 \leq k \leq n$ and $\eta: \tilde{N} \rightarrow\{ \pm 1\}$ be a linear character which takes value 1 on exactly $k$ elements of $\left\{\sigma_{1}, \sigma_{2}, \ldots, \sigma_{n}\right\}$ and value -1 , otherwise. Then the usual construction of $\operatorname{Irr}(\tilde{W})$ via Clifford theory (see [5, 5.5.4]) shows that

$$
\left\langle\tilde{\phi}^{\alpha, \beta}, \operatorname{Ind}_{\tilde{N}}^{\tilde{W}}(\eta)\right\rangle \neq 0 \quad \Longleftrightarrow \quad \alpha \vdash k \text { and } \beta \vdash n-k .
$$

Assume now that $(\alpha, \beta) \vdash n$ is such that $\left\langle\tilde{\phi}^{\alpha, \beta}, \tilde{\rho}_{C}\right\rangle \neq 0$. Let $k=|\alpha|$ and $\eta$ be a linear character of $\tilde{N}$ as above. Then we also have

$$
\left\langle\operatorname{Ind}_{\tilde{N}}^{\tilde{W}}(\eta), \tilde{\rho}_{C}\right\rangle \neq 0 .
$$

Now, by Remark 3.3. $\tilde{\rho}_{C}$ is obtained by inducing a certain linear character from $C_{\tilde{W}}\left(w_{I}\right)$ to $\tilde{W}$. Hence, using the Mackey formula, we conclude that there exists some $x \in \tilde{W}$ such that $\eta^{x}$ and $\tilde{\epsilon}_{I}$ have the same restriction to $\tilde{N} \cap C_{\tilde{W}}\left(w_{I}\right)$. Note that $\eta^{x}$ is a linear character of $\tilde{N}$ similar to $\eta$, that is, it takes value 1 on exactly $k$ elements of $\left\{\sigma_{1}, \sigma_{2}, \ldots, \sigma_{n}\right\}$ and value -1 , otherwise. Now let $\tilde{W}_{I} \subseteq \tilde{W}$ be a parabolic subgroup such that Remark $\underline{3.2}(*)$ holds. Then $\tilde{W}_{I}$ is of type $B_{m} \times A_{1} \times$ $\cdots \times A_{1}$ where $m \geq 0$ and where the number of factors of type $A_{1}$ is strictly smaller than $n / 2$. Consequently, the element $w_{I}$ has the following form:

$$
w_{I}=\sigma_{1} \sigma_{2} \cdots \sigma_{m} s_{m+1} s_{m+3} \cdots s_{m+2 l-1} \quad \text { where } \quad 0 \leq l<n / 2 .
$$

It follows that $\tilde{N} \cap C_{\tilde{W}}\left(w_{I}\right)$ is generated by the elements

$$
\begin{aligned}
& \sigma_{1}, \quad \sigma_{2}, \ldots, \quad \sigma_{m}, \\
& \sigma_{m+1} \sigma_{m+2}, \sigma_{m+3} \sigma_{m+4}, \ldots, \sigma_{m+2 l-1} \sigma_{m+2 l}, \\
& \sigma_{m+2 l+1}, \quad \sigma_{m+2 l+2}, \ldots,
\end{aligned}
$$

(To see this, it may be useful to work with the usual realisation of $\tilde{W}$ as a group of monomial matrices. In this realisation, each $\sigma_{i}$ is represented by a diagonal matrix where the $i$ th diagonal entry is -1 and all other diagonal entries are 1.) By the construction of $\tilde{\epsilon}_{I}$, we have

$$
\begin{aligned}
\tilde{\epsilon}_{I}\left(\sigma_{i}\right) & =1 & & (1 \leq i \leq m), \\
\tilde{\epsilon}_{I}\left(\sigma_{m+2 i-1} \sigma_{m+2 i}\right) & =-1 & & (1 \leq i \leq l), \\
\tilde{\epsilon}_{I}\left(\sigma_{i}\right) & =1 & & (i \geq m+2 l+1) .
\end{aligned}
$$

(The first and third equalities are clear. As for the second equality, note that $\sigma_{m+2 i-1} \sigma_{m+2 i}=s_{m+2 i-1} y$ where $s_{m+2 i-1} \in I$ and $y=\sigma_{m+2 i-1} s_{m+2 i-1} \sigma_{m+2 i-1} \in$ 
$\tilde{Y}$; so, by Remark 3.3. we have $\tilde{\epsilon}_{I}\left(s_{m+2 i-1}\right)=-1$ and $\tilde{\epsilon}_{I}(y)=1$.) Since $\eta^{x}$ and $\tilde{\epsilon}_{I}$ have the same restriction to $\tilde{N} \cap C_{\tilde{W}}\left(w_{I}\right)$, we must have $\eta^{x}\left(\sigma_{i}\right)=1$ for $1 \leq i \leq m$ and $\eta^{x}\left(\sigma_{i}\right)=1$ for $i \geq m+2 l+1$. Furthermore, for each $i \in\{1, \ldots, l\}$, we must have either $\eta^{x}\left(\sigma_{m+2 i-1}\right)=1$ or $\eta^{x}\left(\sigma_{m+2 i}\right)=1$. Hence, $\eta^{x}$ takes value -1 on exactly $l$ elements in $\left\{\sigma_{1}, \sigma_{2}, \ldots, \sigma_{n}\right\}$. Thus, we conclude that $k=n-l>n / 2$, as required.

Recall Lusztig's notion of special characters for $W$ and of the finite 2-group associated to the family of a special character; see [10, Chap. 4].

Proposition 3.5. Let $W=D_{n}(n \geq 2)$ and $\sigma$ the graph automorphism of order 2 , as above. As a class function on $W . \sigma$, we have

$$
\tilde{\rho}=\sum_{(\alpha, \beta)} 2^{d(\alpha, \beta)} \tilde{\phi}^{\alpha, \beta},
$$

where the sum is over all pairs $(\alpha, \beta) \vdash n$ such that $|\alpha|>|\beta|$ and $\phi^{\alpha, \beta}$ is special, and $2^{d(\alpha, \beta)}$ is the order of the finite group attached to the family of $\phi^{\alpha, \beta}$. All the extended characters $\tilde{\phi}^{\alpha, \beta}$ appearing in the above sum are the preferred extensions as in Remark 2.7.

Proof. First note that, in the decomposition of $\tilde{\rho}$ as a class function on $W . \sigma$, there will only be characters $\tilde{\phi}^{\alpha, \beta}$ where $\alpha \neq \beta$. Now, by [6, 3.3], $\phi^{\alpha, \beta}(\alpha \neq \beta)$ is a constituent of $\rho$ if and only if it is a special character, and in that case, the multiplicity is given by $2^{d(\alpha, \beta)}$. In combination with Lemma 3.4 (and Remark 2.5), this immediately yields the above formula for $\tilde{\rho}$.

It remains to check the statement concerning the preferred extensions. Let $(\alpha, \beta) \vdash n$ be such that $\alpha \neq \beta$ and consider the corresponding character $\phi^{\alpha, \beta} \in$ $\operatorname{Irr}(W)$. As in [10,4.6], we have an associated symbol with two rows of equal length

$$
\left(\begin{array}{c}
\lambda_{1}<\lambda_{2}<\ldots<\lambda_{m} \\
\mu_{1}<\mu_{2}<\ldots<\mu_{m}
\end{array}\right)
$$

where the upper row is associated with $\alpha$ and the lower row is associated with $\beta$. Recall from Remark 2.7 that $\tilde{\phi}^{\alpha, \beta} \in \operatorname{Irr}(\tilde{W})$ is the preferred extension of $\phi^{\alpha, \beta}$ if the above symbol has the following property: the smallest entry which appears in only one row appears in the lower row. (Note that $\phi^{\alpha, \beta}=\phi^{\beta, \alpha}$, so the two rows of the symbol can be interchanged as far as $\operatorname{Irr}(W)$ is concerned; however, when we consider extensions to $\tilde{W}$, then $\tilde{\phi}^{\alpha, \beta} \neq \tilde{\phi}^{\beta, \alpha}$ and so the order of the two rows does matter.) Now, by [10, 4.6], the condition for $\phi^{\alpha, \beta}$ to be special is that either

$$
\lambda_{1} \leq \mu_{1} \leq \lambda_{2} \leq \mu_{2} \leq \ldots \leq \lambda_{m} \leq \mu_{m}
$$

or

$$
\mu_{1} \leq \lambda_{1} \leq \mu_{2} \leq \lambda_{2} \leq \ldots \leq \mu_{m} \leq \lambda_{m} .
$$

Hence, if $\phi^{\alpha, \beta}$ is special, then $\tilde{\phi}^{\alpha, \beta}$ is the preferred extension precisely when we are in the second case. But in this case, we have $\sum_{1 \leq i \leq m} \mu_{i}<\sum_{1 \leq i \leq m} \lambda_{i}$ which immediately implies that $|\beta|<|\alpha|$. Thus, all the characters $\tilde{\phi}^{\alpha, \beta}$ appearing in the decomposition of $\tilde{\rho}$ are preferred extensions.

Remark 3.6. Assume that $n$ is odd. Then one can give a different proof based on Proposition 2.6, as follows. We need to specify certain involutions in $W$. For this, recall that $W$ can be realized as a subgroup of index two in the group of signed permutations of $\{1, \ldots, n\}$. For non-negative integers $j, k, l$ with $n=2 j+k+l$ let 
$t_{j, k, l}$ denote the involution in $W$ which acts by the permutation $(1,2) \cdots(2 j-1,2 j)$ on the first $2 j$ letters, and by -1 on the last $l$, and write $C(j, k, l)$ for its class. Then by [6, 3.3], $\phi^{\alpha, \beta}$ occurs in $\rho_{C(j, k, l)}$ if and only if $\alpha \vdash j+k+l, \beta \vdash j$. Now the character $\tilde{\phi}^{\alpha, \beta}$ has sign $(-1)^{|\beta|}$ on the central element $w_{0} \sigma$, while the length of $t_{j, k, l}$ clearly satisfies $l\left(t_{j, k, l}\right) \equiv j(\bmod 2)$, so we may conclude by Proposition 2.6 .

\section{EXCEPtional TyPes}

We now consider the various cases of exceptional type.

4.1. $I_{2}(m), m$ odd, with $o(\sigma)=2$. Let $(W, S)$ be the Coxeter group of type $I_{2}(m), m \geq 3$ odd, and $\sigma$ the graph automorphism of $W$ interchanging the two generating reflections in $S$. Then $\operatorname{Irr}(W)$ consists of the trivial character $\phi_{1,0}$, the sign character $\phi_{1, m}$ and $(m-1) / 2$ irreducible characters $\phi_{2, k}, 1 \leq k \leq(m-1) / 2$, of degree 2 .

Proposition 4.1. Let $W$ be of type $I_{2}(m), m$ odd, and $o(\sigma)=2$. As a class function on $W . \sigma$, we have

$$
\tilde{\rho}=\tilde{\phi}_{1,0}-\tilde{\phi}_{1, m}-\sum_{1 \leq k \leq(m-1) / 2} \tilde{\phi}_{2, k}
$$

where $\tilde{\phi}_{*}$ denotes the extension of $\phi_{*}$ to $W . \sigma$ with positive value on $w_{0} \sigma$.

Proof. By [17, Prop. 3.5] we have

$$
\rho=\sum_{\phi \in \operatorname{Irr}(W)} \phi
$$

According to [17, Tab. 1] all constituents except for $\phi_{1,0}$ occur in $\rho_{C}$, where $C$ is the unique conjugacy class of involutions of $W$. Thus, by Proposition 2.6 $\left\langle\tilde{\rho}_{C}, \tilde{\phi}\right\rangle=$ $\left\langle\rho_{C}, \phi\right\rangle=1$ for all of these, with $\tilde{\phi}$ the extension with $(-1)^{l(t)} \tilde{\phi}\left(w_{0} \sigma\right)>0$, where $t \in C$. Since the generating reflections lie in $C$ and have length 1 , the claim follows.

4.2. $I_{2}(m), m$ even, with $o(\sigma)=2$. Let $(W, S)$ be the Coxeter group of type $I_{2}(m), m \geq 4$ even, and $\sigma$ the exceptional graph automorphism of $W$ interchanging the two generating reflections in $S$. Then $W$ has the trivial character $\phi_{1,0}$, the sign character $\phi_{1, m}$, two further linear characters $\phi_{1, m / 2}^{\prime}$ and $\phi_{1, m / 2}^{\prime \prime}$, and $m / 2-1$ irreducible characters $\phi_{2, k}$. All of these except for the two linear characters $\phi_{1, m / 2}^{\prime}$ and $\phi_{1, m / 2}^{\prime \prime}$ are $\sigma$-invariant.

Proposition 4.2. Let $W$ be of type $I_{2}(m), m$ even, and $o(\sigma)=2$. As a class function on $W . \sigma$, we have

$$
\tilde{\rho}=\tilde{\phi}_{1,0}+\tilde{\phi}_{1, m}
$$

where $\tilde{\phi}_{1, k}$ denotes the extension of $\phi_{1, k}$ to $W . \sigma$ with positive value on $\sigma$, for $k=$ $0, m$.

Proof. First consider the case where $m \equiv 2(\bmod 4)$. Then by [17, Prop. 3.5],

$$
\rho=\phi_{1,0}+\phi_{1, m / 2}^{\prime}+\phi_{1, m / 2}^{\prime \prime}+\phi_{1, m}+\sum_{1 \leq k \leq(m-2) / 4} 2 \phi_{2,2 k-1} .
$$

As pointed out above, the second and third linear character are interchanged by $\sigma$. Moreover, according to [17, Tab. 1] for any $k$ the two copies of $\phi_{2,2 k-1}$ lie 
in submodules corresponding to the involution classes of the two (non-conjugate) elements in $S$, which are interchanged by $\sigma$. Thus the extension of their sum vanishes on $W . \sigma$. Similarly, when $m \equiv 0(\bmod 4)$ then by [17, Prop. 3.5] we have

$$
\rho=\phi_{1,0}+\phi_{1, m}+\sum_{1 \leq k \leq m / 4} 2 \phi_{2,2 k-1} .
$$

The same argument as before applies.

Example 4.3. The above covers in particular the exceptional graph automorphisms of the two Weyl groups of type $B_{2}$ and $G_{2}$.

(1) Let $W$ be of type $B_{2}$ with $\sigma$ interchanging the two generating reflections in $S$. Here $\operatorname{Irr}(W)$ has five elements, among which are the trivial representation $\phi_{1,0}$, the sign representation $\phi_{1,4}$ and the reflection representation $\phi_{2,1}$. We denote by $\tilde{\phi}_{1, k}$ the extension of $\phi_{1, k}$ to $\tilde{W}=\langle W, \sigma\rangle$ taking value 1 on $\sigma$, for $k=0,4$. Then by Proposition 4.2 we have

$$
\tilde{\rho}=\tilde{\phi}_{1,0}+\tilde{\phi}_{1,4} \text {. }
$$

(2) Let $W$ be of type $G_{2}$ with $\sigma$ interchanging the two generating reflections in $S$. Here $\operatorname{Irr}(W)$ has six elements, among which are the trivial representation $\phi_{1,0}$, the sign representation $\phi_{1,6}$, and the reflection representation $\phi_{2,1}$. We denote by $\tilde{\phi}_{1, k}$ the extension of $\phi_{1, k}$ to $\tilde{W}=\langle W, \sigma\rangle$ taking value 1 on $\sigma$, for $k=0,6$. Then by Proposition 4.2 we have

$$
\tilde{\rho}=\tilde{\phi}_{1,0}+\tilde{\phi}_{1,6} .
$$

4.3. $D_{4}$ with $o(\sigma)=3$. Let $(W, S)$ be the Coxeter group of type $D_{4}$, and $\sigma$ the exceptional graph automorphism of $W$ of order 3. Here $\operatorname{Irr}(W)$ has 13 elements, seven of which extend to $\tilde{W}=\langle W, \sigma\rangle$. We denote the irreducible characters of $W$ by pairs of partitions, as in the previous section.

Proposition 4.4. Let $W$ be of type $D_{4}$ and $o(\sigma)=3$. As a class function on $W . \sigma$, we have

$$
\tilde{\rho}=\tilde{\phi}^{-, 4}+\tilde{\phi}^{-, 1^{4}}+\tilde{\phi}^{1,3}+\tilde{\phi}^{1,1^{3}}+2 \tilde{\phi}^{1,21},
$$

where $\tilde{\phi}^{*}$ denotes the extension of $\phi^{*}$ to $W . \sigma$ which takes positive integral value on $\sigma$. (These are the preferred extensions as in Remark 2.7.)

Proof. By [17, Thm. 1.4] the decomposition of $\rho$ is given by

$\rho=\phi^{-, 4}+\phi^{-, 1^{4}}+\phi^{1,3}+\phi^{1,1^{3}}+2 \phi^{1,21}+\phi^{2,+}+\phi^{2,-}+\phi^{1^{2},+}+\phi^{1^{2},-}+\phi^{-, 31}+\phi^{-, 21^{2}}$.

Here, the first five constituents extend to $\tilde{W}$, while the last six are permuted in triples by $\sigma$. Now the values of the extensions $\tilde{\phi}^{*}$ of the first five constituents on $\sigma$ add up to $\tilde{\rho}(\sigma)=8=\left|\mathbf{I}^{\sigma}\right|$, thus these are exactly the constituents of $\tilde{\rho}$ on $W \cdot \sigma$.

4.4. $F_{4}$ with $o(\sigma)=2$. Let $(W, S)$ be the Coxeter group of type $F_{4}$, and $\sigma$ the exceptional graph automorphism of $W$ of order 2. Here $\operatorname{Irr}(W)$ has 25 elements, eleven of which extend to $\tilde{W}=\langle W, \sigma\rangle$.

Proposition 4.5. Let $W$ be of type $F_{4}$ and let $o(\sigma)=2$. As a class function on $W . \sigma$, we have

$$
\tilde{\rho}=\tilde{\phi}_{1,0}+\tilde{\phi}_{9,2}+\tilde{\phi}_{9,10}+\tilde{\phi}_{1,24}+\tilde{\phi}_{12,4}-2 \tilde{\phi}_{6,6}^{\prime \prime}+\tilde{\phi}_{6,6}^{\prime},
$$

where $\tilde{\phi}_{*}$ are the extensions of $\phi_{*}$ printed in [4, Tab. 1]. 
Proof. By the result of Casselman [1, p. 39], the decomposition of $\rho$ is given by

$$
\begin{aligned}
\rho= & \phi_{1,0}+\phi_{9,2}+\phi_{8,3}^{\prime}+\phi_{8,3}^{\prime \prime}+\phi_{8,9}^{\prime}+\phi_{8,9}^{\prime \prime}+\phi_{9,10}+\phi_{1,24}+2 \phi_{4,1}+2 \phi_{4,13} \\
& +3 \phi_{12,4}+\phi_{9,6}^{\prime}+2 \phi_{6,6}^{\prime \prime}+\phi_{9,6}^{\prime \prime}+\phi_{6,6}^{\prime} .
\end{aligned}
$$

Of these, the characters of degree 8 and the characters $\phi_{9,6}^{\prime}, \phi_{9,6}^{\prime \prime}$ do not extend to $\tilde{W}$. By [1, p.39] the two copies of representations $\phi_{4,1}$ and $\phi_{4,13}$ lie in two submodules corresponding to non-conjugate involutions interchanged by $\sigma$, so $W . \sigma$ has trace 0 on their sum. By explicit computation of traces on the submodules of dimensions 18 and 72 corresponding to the two $\sigma$-fixed non-central classes of involutions one finds the stated decomposition.

4.5. $E_{6}$ with $o(\sigma)=2$. Let $(W, S)$ be the Coxeter group of type $E_{6}$, and $\sigma$ the graph automorphism of $W$ of order 2 ; it is given by conjugation with the longest element $w_{0}$. Here $\operatorname{Irr}(W)$ has 25 elements, all of which extend to $\tilde{W}=\langle W, \sigma\rangle$.

Proposition 4.6. Let $W$ be of type $E_{6}$ and let $o(\sigma)=2$. As a class function on $W . \sigma$, we have

$$
\begin{aligned}
\tilde{\rho}= & \tilde{\phi}_{1,0}+\tilde{\phi}_{6,1}+\tilde{\phi}_{20,2}+\tilde{\phi}_{64,4}+\tilde{\phi}_{60,5}+\tilde{\phi}_{81,6}+\tilde{\phi}_{24,6}+\tilde{\phi}_{81,10}+\tilde{\phi}_{60,11}+\tilde{\phi}_{24,12} \\
& +\tilde{\phi}_{64,13}+\tilde{\phi}_{20,20}+\tilde{\phi}_{6,25}+\tilde{\phi}_{1,36}+2 \tilde{\phi}_{30,3}+2 \tilde{\phi}_{30,15}+2 \tilde{\phi}_{80,7}+\tilde{\phi}_{90,8}+\tilde{\phi}_{10,9},
\end{aligned}
$$

where $\tilde{\phi}_{*}$ denotes Lusztig's preferred extension of $\phi_{*}$ to W. $\sigma$ (see Remark 2.7).

Proof. By the result of Casselman [1, p. 40], the decomposition of $\rho$ is given by

$$
\begin{aligned}
\rho= & \phi_{1,0}+\phi_{6,1}+\phi_{20,2}+\phi_{64,4}+\phi_{60,5}+\phi_{81,6}+\phi_{24,6}+\phi_{81,10}+\phi_{60,11}+\phi_{24,12} \\
& +\phi_{64,13}+\phi_{20,20}+\phi_{6,25}+\phi_{1,36}+2 \phi_{30,3}+2 \phi_{30,15}+2 \phi_{80,7}+\phi_{90,8}+\phi_{10,9} .
\end{aligned}
$$

So the claim follows from Proposition 2.6 and inspection of the Chevie [18] tables of $W$ and $\tilde{W}$.

Remark 4.7. Assume that $W$ is an irreducible Weyl group and $\sigma$ is ordinary (see Remark 2.7). Then the results in this and the previous section show that, in the decomposition of $\tilde{\rho}$ as a class function on $W . \sigma$, all multiplicities are $\geq 0$ if we choose Lusztig's preferred extensions of the characters in $\operatorname{Irr}(W)^{\sigma}$ as in Remark 2.7 .

\section{Frobenius-Schur indicAtors AND Fourier Matrices}

We shall now interpret the multiplicity formulae for $\tilde{\rho}$ obtained in the previous two sections in terms of Fourier matrices.

5.1. The Fourier matrix associated with $W, \sigma$ is a matrix with rows and columns labelled by two finite, purely combinatorially defined sets $\bar{X}(W, \sigma)$ and $\Lambda(W, \sigma)$, respectively. We have $|\bar{X}(W, \sigma)|=|\Lambda(W, \sigma)|$ and there is a well-defined injection $\operatorname{Irr}(W)^{\sigma} \hookrightarrow \Lambda(W, \sigma)$. Let us briefly recall how these are defined.

If $W$ is a Weyl group and $\sigma$ is ordinary (see Remark 2.7), then $\bar{X}(W, \sigma)$ is the set defined in [10,4.21.11], which is in bijection with the set of unipotent characters of a corresponding finite group of Lie type by [10, Main Theorem 4.23]. The set $\Lambda(W, \sigma)$ is obtained by choosing a complete set of representatives for the $M$-orbits on the set $X(W, \sigma)$ defined in [10, 4.21.12] (with $M$ as in [10, 4.16]). Then [10, 4.21.14] gives rise to the natural injection $\operatorname{Irr}(W)^{\sigma} \hookrightarrow \Lambda(W, \sigma)$. The corresponding Fourier matrix is obtained as follows. The entry for $\bar{x} \in \bar{X}(W, \sigma)$ and $y \in \Lambda(W, \sigma)$ is given by evaluating the canonical pairing $\bar{X}(W, \sigma) \times X(W, \sigma) \rightarrow \overline{\mathbb{Q}}_{l}$ in [10, 4.21.13] 
on $(\bar{x}, y)$ and then multiplying the result by a sign $\Delta(\bar{x})= \pm 1$, as defined in [10, pp. 124-126]. If $W$ is not a Weyl group, or if $W$ is of type $B_{2}, G_{2}$ or $F_{4}$ and $\sigma$ is not ordinary, the Fourier matrix has been described by heuristic methods in 12 ] and 4, respectively.

Now, if $\sigma=\mathrm{id}$, there is a canonical identification $\Lambda(W, \sigma)=\bar{X}(W$, id $)$, hence the Fourier matrix is canonically defined in this case. Otherwise, there are certain choices involved in the definition of the Fourier matrix. As far as the entries corresponding to the image of $\operatorname{Irr}(W)^{\sigma}$ in $\Lambda(W, \sigma)$ are concerned, these depend precisely on the choices of extensions of the characters in $\operatorname{Irr}(W)^{\sigma}$ to the coset $W . \sigma$.

The following result provides the promised interpretation of the decomposition of $\tilde{\rho}$ in the case where $W$ and $\sigma$ arise from a simple algebraic group $G$ and an endomorphism $F: G \rightarrow G$ as in Section 1 In this case, the Fourier matrix describes the multiplicities

$$
\left\langle\chi, R_{\tilde{\phi}}\right\rangle \quad \text { where } \quad R_{\tilde{\phi}}:=\frac{1}{|W|} \sum_{w \in W} \tilde{\phi}(w \sigma) R_{T_{w}, 1},
$$

for any $\chi \in \operatorname{Uch}\left(G^{F}\right)$ and $\phi \in \operatorname{Irr}(W)^{\sigma}$; see [10, Main Theorem 4.23].

Theorem 5.1. Let $W$ be a finite irreducible Weyl group with generating set $S$, and $\sigma: W \rightarrow W$ a non-trivial automorphism with $\sigma(S)=S$. For each $\phi \in \operatorname{Irr}(W)^{\sigma}$, we fix an extension $\tilde{\phi}$ to W. $\sigma$. Then the $\{1,-1,0\}$-vector of Frobenius-Schur indicators of the unipotent characters of the corresponding twisted groups of Lie type (indexed by the set $\bar{X}(W, \sigma))$ is mapped under Fourier transform and restriction to the image of $\operatorname{Irr}(W)^{\sigma}$ in $\Lambda(W, \sigma)$ onto the vector of multiplicities in the decomposition of $\tilde{\rho}$ as a class function on $W . \sigma$.

Proof. First note that the assertion does not depend on the actual choices of the extensions $\tilde{\phi}$, as long as we use the same extensions both for the Fourier matrix and for the decomposition of $\tilde{\rho}$. Now we consider the various cases.

First, let $W=\mathfrak{S}_{n}$ with the non-trivial graph automorphism $\sigma$. We have a natural parametrisation $\operatorname{Uch}\left(\mathrm{GU}_{n}(q)\right)=\left\{\chi_{\alpha} \mid \alpha \vdash n\right\}$ where the degree of $\chi_{\alpha}$ is given in terms of a well-defined polynomial in $q$; see Lusztig [8, $\S 9$ ] for further details. Let $A_{\alpha}$ denote the degree in $q$ of the degree polynomial of $\chi_{\alpha}$; its order of vanishing at $q=0$ is the invariant $a_{\alpha}$ already mentioned in Remark 2.7. We claim that

(†) $\chi_{\alpha}$ has Frobenius-Schur indicator $(-1)^{a_{\alpha}+A_{\alpha}}$.

This is seen as follows. By Ohmori [19] (see also [13]), the Frobenius-Schur indicator of $\chi_{\alpha}$ is given by $(-1)^{\lfloor k / 2\rfloor}$ where $\kappa$ is the 2-core of $\alpha$. Now $\chi_{\alpha}$ lies in the HarishChandra series of the (unique) cuspidal unipotent character $\lambda$ of $\mathrm{GU}_{k}(q)$ (see 8 , 9.6]). In particular ([8,7.8]), its degree polynomial is divisible by the same power of $q-1$ as that of $\lambda$, viz. $q-1$ to the power $\lfloor k / 2\rfloor$. Now note that unipotent characters of general linear groups all lie in the principal series, so their degree polynomials are not divisible by $q-1$; thus, by Ennola duality (see [8, 9.5]) the degree polynomials of unipotent characters of unitary groups are not divisible by $q+1$. So the only odd degree factors of these degree polynomials are $q$ and $q-1$. In particular, we obtain $A_{\alpha} \equiv a_{\alpha}+\lfloor k / 2\rfloor(\bmod 2)$. Thus $(\dagger)$ is proved. Now consider the corresponding Fourier matrix. Since all families are singletons, this matrix is diagonal with \pm 1 on the diagonal. By the description of the pairing 
$\bar{X}(W, \sigma) \times \Lambda(W, \sigma)$ in [10, 4.19] and that of the $\Delta$-function in [10, p. 124] (see also p. 235 in the proof of [10, Prop. 7.6]), we have $\left\langle\chi_{\alpha}, R_{\tilde{\phi}_{\alpha}}\right\rangle=(-1)^{A_{\alpha}}$ where $\tilde{\phi}_{\alpha}$ is the extension of $\phi_{\alpha}$ in which $\sigma$ acts as the longest element $w_{0}$. Passing to the preferred extension, we conclude that the corresponding diagonal entry of the Fourier matrix is $(-1)^{a_{\alpha}+A_{\alpha}}$. Hence, multiplication with the Fourier matrix indeed gives the multiplicities computed in Proposition 3.1

In Type $D_{n}, o(\sigma)=2$, all Frobenius-Schur indicators of the unipotent characters of ${ }^{2} D_{n}(q)$ are equal to +1 by Lusztig [13, 1.13]. The Fourier matrix is described in [10, 4.18]. It is a block diagonal matrix with blocks corresponding to the various $\sigma$-stable families of $\operatorname{Irr}(W)$. It is clear that all the 1 -vector transforms to the vector with value $\pm 2^{d}$ at the image of a $\sigma$-stable special character in $\Lambda(W, \sigma)$ (where $2^{d}$ is the order of the finite group associated with the family containing the given special character) and 0 otherwise. We need to show that, if we choose preferred extensions as in Proposition 3.5, then the above values are always $+2^{d}$. For this purpose, it will be enough to show that the entries in the Fourier matrix corresponding to the preferred extension of a $\sigma$-stable special character are all $\geq 0$. But this follows from the description in [10, 4.18]. Indeed, let $\phi \in \operatorname{Irr}(W)$ be a $\sigma$-stable special character. As in [10,4.6], we have a corresponding nondegenerate symbol $Z$ with two rows of equal length. Let $Z_{1}$ be the set of singles in $Z$ and let $Z_{2}$ be the set of doubles in $Z$. Furthermore, let $Z_{1}=M_{0} \amalg M_{0}^{\prime}$ be the partition defined by the two rows of $Z$, where $M_{0}, M_{0}^{\prime}$ are distinguished one from another by the inequality $\sum_{x \in M_{0}} x<\sum_{x \in M_{0}^{\prime}} x$, as in [10, p. 93]. Then, as in [10, p. 117], the two extensions of $\phi$ are labelled by the symbols

$$
\left(\begin{array}{c}
Z_{2} \amalg M_{0}^{\prime} \\
Z_{2} \amalg M_{0}
\end{array}\right) \quad \text { and } \quad\left(\begin{array}{c}
Z_{2} \amalg M_{0} \\
Z_{2} \amalg M_{0}^{\prime}
\end{array}\right) \text {, }
$$

respectively. Now recall that a preferred extension is characterised by the condition that the smallest element of $Z_{1}$ has to appear in the lower row of the symbol associated with the extension. Hence, using an argument as in the proof of Proposition 3.5, it immediately follows that the preferred extension of a special character is labelled by the first of the above two symbols (the one where $M_{0}$ is in the lower row). The formula at the end of $[10,4.18]$ then shows that the corresponding entries in the Fourier matrix are all $\geq 0$, as required. Thus, we obtain the vector of multiplicities in $\tilde{\rho}$ as computed in Proposition 3.5.

For $W=E_{6}$ with $\sigma$ the non-trivial graph automorphism, it is shown in 3 , $5.6,6.5]$ that all unipotent characters of ${ }^{2} E_{6}(q)$ have Frobenius-Schur indicator +1 , except for the two cuspidal unipotent characters denoted ${ }^{2} E_{6}[\theta],{ }^{2} E_{6}\left[\theta^{2}\right]$ where the indicator is 0 , and for the two characters lying above the cuspidal unipotent character of $\mathrm{GU}_{6}(q)$, with indicator -1 . The Fourier matrices are described in [9, Thm. 1.15] (see also [10, Prop. 7.11]), with respect to the extensions of the $\sigma$ stable characters of $W$ which have positive value on $w_{0} \sigma$. Let $E_{6}(q)$ be the Chevalley group of split type $E_{6}$. Then there is a bijection $\chi \leftrightarrow \chi^{\prime}$ between $\operatorname{Uch}\left({ }^{2} E_{6}(q)\right)$ and $\mathrm{U} \operatorname{ch}\left(E_{6}(q)\right)$ such that

$$
\left\langle\chi, R_{\tilde{\phi}}\right\rangle=\delta_{\chi}\left\langle\chi^{\prime}, R_{\phi}\right\rangle \quad \text { for all } \chi \in \mathrm{U} \operatorname{ch}\left({ }^{2} E_{6}(q)\right) \text { and } \phi \in \operatorname{Irr}(W)^{\sigma},
$$

where $\delta_{\chi}= \pm 1$ is determined by the condition that the degree polynomial of $\chi$ is obtained from that of $\chi^{\prime}$ by formally replacing $q$ by $-q$ and then multiplying by $\delta_{\chi}$. Hence, using the Fourier matrix for $E_{6}(q)$ and the table of degree polynomials 
in [10, p. 363], and then passing to the preferred extensions, the claim follows by comparison with Proposition 4.6.

For $W=D_{4}, o(\sigma)=3$, the corresponding finite reductive group ${ }^{3} D_{4}(q)$ has five families of unipotent characters, with 1,1,4,1,1 characters, respectively (see [9, 1.17]), and all Frobenius-Schur indicators are equal to 1 (see [3, Tab. 1]). The Fourier matrix (see [9, Thm. 1.18] or [10, Prop. 7.6]) transforms this to the vector $(1,1,2,0,0,0,1,1)$, which upon comparing the labels, just gives the decomposition in Proposition 4.4.

Now consider the cases where $\sigma$ is not ordinary. First, let $W=B_{2}, o(\sigma)=2$. There are three families of unipotent characters for the corresponding finite reductive group ${ }^{2} B_{2}\left(q^{2}\right)$, with 1, 2, 1 characters, respectively (see e.g. [4, 2.3]), and Frobenius-Schur indicators 1,0,0,1. The transform under the Fourier matrix is thus $(1,0,0,1)$, where the entries equal to 1 correspond to the class functions $\tilde{\phi}_{1,0}, \tilde{\phi}_{1,4}$ on $W . \sigma$, respectively. By Proposition 4.2 this is the vector of multiplicities in $\tilde{\rho}$, as claimed.

For $W=G_{2}, o(\sigma)=2$, the corresponding finite reductive group ${ }^{2} G_{2}\left(q^{2}\right)$ has three families of unipotent characters, with 1,6,1 characters, respectively, and Frobenius-Schur indicators $1,0,0,0,0,0,0,1$. This is an eigenvector of the Fourier transform matrix given in [4, Thm. 5.4], and the claim follows with Proposition 4.2.

For $W=F_{4}, o(\sigma)=2$, the corresponding finite reductive group ${ }^{2} F_{4}\left(q^{2}\right)$ has seven families of unipotent characters, with $1,1,1,1,2,2,13$ characters, respectively. The Frobenius-Schur indicators in the 1-element families are equal to 1, in the 2-element families equal to 0 , and on the 13-element family are given by $(1,1,1,1,1,1,0,0,0$, $0,0,0,-1)$ (see [3, §7]). Multiplication with the Fourier transform matrix [4, Tab. 2] here gives the vector $(1,0,1,-2,0,0,0,0,-1,0,0,0,0)$, which upon comparing labels with loc. cit. gives the claim by Proposition 4.5.

Remark 5.2. Lusztig [7, 3.9] has shown that one can attach to each $\chi \in \operatorname{Uch}\left(G^{F}\right)$ a corresponding "eigenvalue of Frobenius", which is a root unity and will be denoted by $\operatorname{Fr}(\chi)$. The results on character fields for unipotent characters in [3, $\S 5]$ and [4. §4] show that, for any $\chi \in \operatorname{Uch}\left(G^{F}\right)$, we have

$$
\nu(\chi)=0 \quad \Longleftrightarrow \quad \operatorname{Fr}(\chi) \text { is non-real. }
$$

Under the Fourier matrix, the unipotent characters transform to a new set of class functions which are labelled by $\Lambda(W, \sigma)$; see [10, 4.24.1]. These class functions are called "almost characters" of $G^{F}$. One can also attach a Frobenius eigenvalue to any such almost character (by using "twisting operators"; see [4, §5] and the references there).

Now, it is not true in general that the vector of Frobenius-Schur indicators, multiplied by the Fourier-transform, has non-zero values only on the image of $\operatorname{Irr}(W)^{\sigma}$ in $\Lambda(W, \sigma)$. For example, in type $F_{4}$, we also find non-zero values for $F_{4}^{I}[1]$; in type $E_{8}$ for $E_{8}^{I}[1]$ (recall that, in the split case, we can identify $\bar{X}(W, \sigma)=\Lambda(W, \mathrm{id})$ ); and in ${ }^{2} F_{4}$ for the element of $\Lambda(W, \sigma)$ denoted $\Psi_{4}$ in [4, Tab. 2]. We would like to point out that in each case, this is one out of the two "cuspidal" elements in $\Lambda(W, \sigma)$ with attached Frobenius eigenvalue 1 .

We are not aware of any, even heuristical, explanation of this. 
5.2. As in [16, §6.4], we shall now reformulate Theorem 5.1] without reference to Fourier matrices. One advantage of this reformulation will be that it does not involve choices of extensions of $\sigma$-stable characters of $W$. We will also be able to drop the assumption that $W$ is irreducible. Let $G$ be a connected reductive algebraic group over $\overline{\mathbb{F}}_{p}$ and let $F: G \rightarrow G$ be an endomorphism as in Section 1, Recall the definitions of $\operatorname{Uch}\left(G^{F}\right)$ and $\mathrm{CF}_{0}\left(G^{F}\right)$. The map $F$ induces an automorphism $\sigma: W \rightarrow W$ such that $\sigma(S)=S$. Let $\tilde{W}=W \rtimes\langle\sigma\rangle$. For any class function $f$ on the coset $W . \sigma$, we define

$$
R_{f}:=\frac{1}{|W|} \sum_{w \in W} f(w \sigma) R_{T_{w}, 1} .
$$

With this notation, we can now state:

Corollary 5.3 (Cf. Lusztig-Vogan [16, 6.4(b)] for the case $\sigma=\mathrm{id}$ ). Let $\tilde{\rho}$ be the character of the extended involution module, as in Section 2. Then

$$
R_{\tilde{\rho}}=\left(\sum_{\chi \in \operatorname{Uch}\left(G^{F}\right)} \nu(\chi) \chi\right)_{0}
$$

where $\nu(\chi)$ denotes the Frobenius-Schur indicator of $\chi$.

Proof. By [2, Prop. 7.10], the set $\mathrm{U} \operatorname{ch}\left(G^{F}\right)$ is "insensitive" to the centre of $G$, and similarly for $R_{f}$. Hence, we may assume, without loss of generality, that $G$ is semisimple of adjoint type. We now proceed by induction on $\operatorname{dim} G$, where we use a reduction argument analogous to [10,8.8].

If $G$ itself is simple, then the result follows from Theorem 5.1. exactly as in [16. 6.4]. Next assume that we have a non-trivial factorisation $G=G_{1} \times G_{2}$ where both $G_{1}$ and $G_{2}$ are $F$-stable. Then $G^{F}=G_{1}^{F} \times G_{2}^{F}$ and the result easily follows by induction. It remains to consider the following case: we have $G=G_{1} \times \cdots \times G_{1}$ (say, $d \geq 2$ factors) where $G_{1}$ is simple of adjoint type; furthermore, $F$ cyclicly permutes the factors and $F^{d}\left(G_{1}\right)=G_{1}$. We then have a natural isomorphism $G^{F} \cong G_{1}^{F^{d}}$ which preserves unipotent characters. Hence, the right-hand side of the desired equality is preserved under this isomorphism. It remains to see what happens on the left-hand side. By the definition of $R_{\tilde{\rho}}$, this is easily reduced to a question about the extended involution module, purely on the level of $W$ and $\sigma$. Now, we have $W=W_{1} \times \cdots \times W_{1}$ (where $W_{1}$ is the Weyl group of $G_{1}$ ) and $\sigma$ cyclicly permutes the factors such that $\sigma^{d}\left(W_{1}\right)=W_{1}$. We need to compare the characters $\tilde{\rho}$ of the extended involution module for $W, \sigma$ and $\tilde{\rho}_{1}$ of the one for $W_{1}, \sigma^{d}$, respectively.

We may assume that for $\left(w_{1}, \ldots, w_{d}\right) \in W_{1} \times \cdots \times W_{1}=W$ we have $\sigma\left(w_{1}, \ldots, w_{d}\right)$ $=\left(w_{2}, \ldots, w_{d}, \sigma^{d}\left(w_{1}\right)\right)$. The map

$$
p: W \rightarrow W_{1}, \quad\left(w_{1}, \ldots, w_{d}\right) \mapsto w_{1} \cdots w_{d},
$$

is surjective with all fibres of size $\left|W_{1}\right|^{d-1}$, and sends $\sigma$-conjugacy classes in $W$ to $\sigma^{d}$-conjugacy classes in $W_{1}$.

Now note that the above isomorphism $G^{F} \cong G_{1}^{F^{d}}$ sends $R_{T_{w}, 1}$ to $R_{T_{p(w)}, 1}$. Thus, it remains to show that $\tilde{\rho}(w \sigma)=\tilde{\rho}_{1}\left(p(w) \sigma^{d}\right)$ for all $w \in W$. Clearly, it is sufficient to check this for class representatives, say on elements of the form $w=\left(w_{1}, 1, \ldots, 1\right)$, where $p(w)=w_{1}$. The only terms contributing to the trace $\tilde{\rho}(w \sigma)$ come from basis elements $a_{t}(t \in \mathbf{I})$ with $w \sigma \cdot a_{t}= \pm a_{t}$, that is, with $w \sigma(t) w^{-1}=t$, whence 
$t \in C_{W}(w \sigma)$. Similarly, contributions to $\tilde{\rho}_{1}\left(w_{1} \sigma^{d}\right)$ come from elements $a_{t_{1}}$ with $t_{1} \in \mathbf{I}_{1} \cap C_{W_{1}}\left(w_{1} \sigma^{d}\right)$. Now

$$
C_{W}(w \sigma)=\left\{\left(g, \sigma^{d}(g), \ldots, \sigma^{d}(g)\right) \mid g \in C_{W_{1}}\left(w_{1} \sigma^{d}\right)\right\},
$$

inducing a natural bijection

$$
\mathbf{I} \cap C_{W}(w \sigma) \stackrel{1-1}{\longrightarrow} \mathbf{I}_{1} \cap C_{W_{1}}\left(w_{1} \sigma^{d}\right), \quad t=\left(t_{1}, \sigma^{d}\left(t_{1}\right), \ldots, \sigma^{d}\left(t_{1}\right)\right) \mapsto t_{1} .
$$

But it is clear from the definition of the involution module that

$$
\left(w_{1}, 1, \ldots, 1\right) \sigma \cdot a_{t}=\left(w_{1}, 1, \ldots, 1\right) a_{\left(\sigma^{d}\left(t_{1}\right), \ldots, \sigma^{d}\left(t_{1}\right)\right)}
$$

and $w_{1} \sigma^{d} \cdot a_{t_{1}}=w_{1} \cdot a_{\sigma^{d}\left(t_{1}\right)}$ have the same sign.

5.3. Finally, as in [17, we now formally define Frobenius-Schur indicators for the combinatorially introduced unipotent characters of dihedral groups with non-trivial automorphism to obtain an analogue of Theorem 5.1 in this case and show a unicity statement.

For this recall that there is a way to attach a set $\operatorname{Uch}\left({ }^{2} I_{2}(m)\right)$ of combinatorial objects, called "unipotent characters", to the dihedral groups $I_{2}(m)(m \geq 3)$ with nontrivial Coxeter automorphism of order 2 (see [12]), such that each $\chi \in \operatorname{Uch}\left({ }^{2} I_{2}(m)\right.$ ) has a degree $\chi(1) \in \mathbb{C}[q]$, and a Frobenius eigenvalue $\operatorname{Fr}(\chi)$ (a root of unity). Following [17] in the untwisted case, we propose to introduce Frobenius-Schur indicators $\nu(\chi)$ satisfying the following properties:

(1) $\nu(\chi) \in\{0, \pm 1\}$

(2) $\nu(\chi)=0$ if and only if the Frobenius eigenvalue $\operatorname{Fr}(\chi)$ is non-real.

The set $\mathrm{U} \operatorname{ch}\left({ }^{2} I_{2}(m)\right)$ is subdivided into three families, two with one element and one containing all the other characters; see [4, 6.1] where one can also find the corresponding Fourier matrix. The characters in the 1-element families have Frobenius eigenvalue 1 and we set $\nu(\chi)=1$ for these.

The unipotent characters in the big family are parametrized by pairs $(k, l)$ of odd integers with $0<k<l<k+l<2 m$, with corresponding Frobenius eigenvalue given by $\zeta^{k l}$, where $\zeta$ is a $2 m$ th root of unity. First assume that $m$ is even. Then $\zeta^{k l}$ is never real, so by (2) above we necessarily have $\nu(\chi)=0$ for all unipotent characters in this big family. Now assume that $m$ is odd. Then certainly $\zeta^{k l}$ is real when $l=m$. We propose to define the Frobenius-Schur indicator $\nu(\chi)$ in this case to be +1 if $l=m$, and 0 otherwise. With this, we have the following extension of [17, Thm. 1.2]:

Theorem 5.4. Let $W=I_{2}(m)$ and $\sigma$ the non-trivial Coxeter automorphism. Then the vector $(\nu(\chi))$ of Frobenius-Schur indicators of unipotent characters defined above is mapped under Fourier transform and restriction to the image of $\operatorname{Irr}(W)^{\sigma}$ in $\Lambda(W, \sigma)$ onto the vector of multiplicities in the decomposition of $\tilde{\rho}$ as a class function on $W . \sigma$.

Moreover, for any finite Coxeter group with non-trivial automorphism, the vector of Frobenius-Schur indicators is the only $\{1,-1,0\}$-vector with this property and satisfying (1), (2) above.

Proof. For the 1-element families, the statement is trivially true, since the corresponding Fourier matrix is the identity matrix and the multiplicities in $\tilde{\rho}$ all equal 1 by Propositions 4.1 and 4.2. When $m$ is odd, note that the data for ${ }^{2} I_{2}(m)$ are Ennola dual to those for $I_{2}(m)$, that is, the Fourier matrix is identical in both cases, 
and the rows and columns are labelled by the same combinatorial objects. The claim then follows by the computation in [17, p. 27]. Finally, when $m$ is even, the first claim is obvious, since both sides are the all 0 -vector. Unicity follows by a combinatorial argument as in [17, p. 27].

For the Weyl groups with non-trivial automorphism, the unicity is clear in the case of 1-element families. For type ${ }^{2} D_{n}$, uniqueness follows exactly as in [17, and for the three exceptional types ${ }^{2} E_{6},{ }^{3} D_{4}$ and ${ }^{2} F_{4}$, it is easily checked by computer.

\section{REFERENCES}

[1] Bill Casselman, Verifying Kottwitz' conjecture by computer, Represent. Theory 4 (2000), 32-45 (electronic), DOI 10.1090/S1088-4165-00-00052-2. MR.1740179 (2000k:20059)

[2] P. Deligne and G. Lusztig, Representations of reductive groups over finite fields, Ann. of Math. (2) 103 (1976), no. 1, 103-161. MR0393266 (52 \#14076)

[3] Meinolf Geck, Character values, Schur indices and character sheaves, Represent. Theory 7 (2003), 19-55 (electronic), DOI 10.1090/S1088-4165-03-00170-5. MR1973366 (2004f:20020)

[4] Meinolf Geck and Gunter Malle, Fourier transforms and Frobenius eigenvalues for finite Coxeter groups, J. Algebra 260 (2003), no. 1, 162-193, DOI 10.1016/S0021-8693(02)00631-2. Special issue celebrating the 80th birthday of Robert Steinberg. MR1973582 (2005d:20067)

[5] Meinolf Geck and Götz Pfeiffer, Characters of finite Coxeter groups and Iwahori-Hecke algebras, London Mathematical Society Monographs. New Series, vol. 21, The Clarendon Press Oxford University Press, New York, 2000. MR.1778802 (2002k:20017)

[6] Robert E. Kottwitz, Involutions in Weyl groups, Represent. Theory 4 (2000), 1-15 (electronic), DOI 10.1090/S1088-4165-00-00050-9. MR1740177 (2000m:22014)

[7] George Lusztig, Representations of finite Chevalley groups, CBMS Regional Conference Series in Mathematics, vol. 39, American Mathematical Society, Providence, R.I., 1978. Expository lectures from the CBMS Regional Conference held at Madison, Wis., August 8-12, 1977. MR.518617 (80f:20045)

[8] G. Lusztig, Irreducible representations of finite classical groups, Invent. Math. 43 (1977), no. 2, 125-175. MR0463275 (57 \#3228)

[9] George Lusztig, On the unipotent characters of the exceptional groups over finite fields, Invent. Math. 60 (1980), no. 2, 173-192, DOI 10.1007/BF01405152. MR586426 (82c:20081)

[10] George Lusztig, Characters of reductive groups over a finite field, Annals of Mathematics Studies, vol. 107, Princeton University Press, Princeton, NJ, 1984. MR742472 (86j:20038)

[11] George Lusztig, Character sheaves. IV, Adv. in Math. 59 (1986), no. 1, 1-63, DOI 10.1016/0001-8708(86)90036-8. MR825086 (87m:20118b)

[12] George Lusztig, Exotic Fourier transform, Duke Math. J. 73 (1994), no. 1, 227-241, 243248, DOI 10.1215/S0012-7094-94-07309-2. With an appendix by Gunter Malle. MR.1257284 (95c:20059)

[13] G. Lusztig, Rationality properties of unipotent representations, J. Algebra 258 (2002), no. 1, 1-22, DOI 10.1016/S0021-8693(02)00514-8. Special issue in celebration of Claudio Procesi's 60th birthday. MR1958895 (2004e:20017)

[14] G. Lusztig, A bar operator for involutions in a Coxeter group. Bull. Inst. Math. Acad. Sinica (N.S.) 7 (2012), 355-404.

[15] G. Lusztig, Asymptotic Hecke algebras and involutions. Preprint, arXiv:1204.0276v1.

[16] G. Lusztig And D. Vogan, Hecke algebras and involutions in Weyl groups. Bull. Inst. Math. Acad. Sinica (N.S.) 7 (2012), 323-354.

[17] E. Marberg, How to compute the Frobenius-Schur indicator of a unipotent character of a finite Coxeter system. Preprint, arXiv:1202.1311.

[18] J. Michel, Development version of the GAP-part of CHEVIE. Webpage and GAP3 package; see http://people.math.jussieu.fr/ jmichel/chevie/chevie.html

[19] Zyozyu Ohmori, The Schur indices of the cuspidal unipotent characters of the finite unitary groups, Proc. Japan Acad. Ser. A Math. Sci. 72 (1996), no. 6, 111-113. MR1404484 (97i:20018) 
Institute of Mathematics, Aberdeen University, Aberdeen AB24 3UE, Scotland, UK. Current address: IAZ - Lehrstuhl für Algebra, Universität Stuttgart, Pfaffenwaldring 57, 70569 Stuttgart, Germany

E-mail address: meinolf.geck@mathematik.uni-stuttgart.de

FB Mathematik, TU Kaiserslautern, Postfach 3049, 67653 Kaiserslautern, Germany.

E-mail address: malle@mathematik.uni-kl.de 